\title{
Una lucha interminable: la tuberculosis en Baja California, México, durante el siglo xx
}

\section{An Endless Fight: Tuberculosis in Baja California, Mexico, During the 20th Century}

\author{
Arturo Fierros Hernández \\ (D) https://orcid.org/0000-0002-5689-7529 \\ Secundaria General núm. 31, Tijuana, Baja California, México. \\ arturofierrosh@gmail.com
}

Resumen: El presente texto es un análisis histórico de la tuberculosis en Baja California durante el siglo $\mathrm{xx}$, en el que se estudia la enfermedad como un proceso aún vigente. Aunque los adelantos en la biomedicina y la salud pública han generado avances en el combate contra la tuberculosis, en la actualidad Baja California ocupa uno de los primeros lugares de incidencia de tal enfermedad. A pesar de que se han identificado las determinantes sociales, así como de que existe un tratamiento efectivo y una vacuna inmunizadora, la lucha contra ese flagelo está lejos de terminar. En este artículo se esbozan las características que podría tener un plan para la erradicación de la enfermedad, un conocimiento útil para entender las epidemias y los sistemas sanitarios, como mostramos a continuación. Este texto es un primer acercamiento a la temática.

Palabras clave: tuberculosis; Baja California; salud pública; endemia; vacuna BCG.

cómo citar: Fierros Hernández, A. (2021). Una lucha interminable: La tuberculosis en Baja California, México, durante el siglo xx. Secuencia (110), e1794. DoI: https://doi.org/10.18234/secuencia.v0i110.1794

cc $(1)$ Esta obra está protegida bajo una Licencia Creative Commons Atribución-NoComercial 4.0 Internacional. 
Abstract: This text is a historical analysis of tuberculosis in Baja California during the 20th century, in which the disease is studied as a current phenomenon. Although advances in biomedicine and public health have made strides in the fight against tuberculosis, Baja California is currently one of the states with the highest incidence of this disease. Although the social determinants have been identified, and effective treatment and an immunizing vaccine exist, the fight against this scourge is far from over. This article outlines the characteristics of a possible plan to eradicate this disease, which is useful for understanding epidemics and health systems, as we show below. This text is a first approach to the subject.

Keywords: tuberculosis; Baja California; public health; endemic; vaccine всG.

Recibido: 11 de octubre de 2019 Aceptado: 21 de mayo de 2020

Publicado: 5 de mayo de 2021

A ti libélula PE

\section{INTRODUCCIÓN}

a tuberculosis en Baja California, a través del tiempo, ha sobrepasado las pues las múltiples acciones sanitarias -que han incluido intervenciones directas e indirectas- impuestas por las más heterogéneas instituciones (nacionales o internacionales) dedicadas directa e indirectamente al cuidado de la salud pública, han sido poco efectivas (Fierros, 2014; Gruel, 2017). Claro es que las acciones sanitarias no son menos importantes que el transcurso mismo de la enfermedad. Entonces subyace la pregunta planteada por Ana María Carrillo (2012, p. 94), ¿la tuberculosis es una enfermedad erradicable?

La misma autora ha analizado la historia de esta enfermedad en su artículo titulado: "Los modernos minotauro y Teseo: la lucha contra la tuberculosis en México" (2012). Ella examina tres momentos que, indica, fueron "fundamentales" en el combate contra la tuberculosis. El primero lo enmarca durante el gobierno de Porfirio Díaz (1876-1910), el segundo en el de Lázaro Cárdenas (1934-1940) y, finalmente, analiza el de Adolfo López Mateos (1958- 
1964). Nos enseña cómo la lucha contra la llamada peste blanca ha tenido claroscuros y se ha visto influenciada por los avances de la ciencia, por supuesto, pero también por creencias y otras situaciones relacionadas con el accionar de los distintos órdenes de gobierno, que han tenido influjo de alguna u otra manera en la salud pública. Pero, sobre todo, nos muestra cómo una enfermedad ha persistido por años en México, no importando las barreras que han impuesto los diversos regímenes políticos que han estado en el poder. El enfoque de Carrillo orbita en lo que Diego Armus (2010) llama: historia de la salud pública, que viene a ser primordialmente una historia enfocada en el poder, la política, el Estado, las instituciones y la profesión médica, en la que de manera muy clara "las relaciones entre las instituciones de salud y las estructuras económicas, sociales y políticas están en el centro de la narrativa” (p. 7).

Asimismo, desde la perspectiva de la historia de la salud pública, Claudia Agostoni ha analizado la tuberculosis en su artículo titulado: "'Timbres rojos' y el Comité Nacional de Lucha contra la Tuberculosis, Ciudad de México, 1939-1950" (2017). En él muestra cómo se han utilizado los avances de la biomedicina en la aplicación pública. Siguiendo igualmente a Armus (2010), discute los problemas de la salud grupal y estudia las acciones políticas para preservar o restaurar la salud colectiva. Señala también los multifactoriales elementos, las condiciones necesarias que propiciaron el desarrollo de la enfermedad y particularmente la puesta en marcha de una estrategia para obtener recursos y concientizar a la población en la lucha contra la tuberculosis. Centra su interés, en consonancia con Armus (2010), en situaciones en que las políticas estatales evalúan tanto factores epidemiológicos, como "políticos, económicos, culturales, científicos y tecnológicos” (p. 7).

En un texto de reciente aparición, Agostoni (2019), desde la óptica de la historia de la enfermedad, ${ }^{1}$ analiza los diversos discursos que se generaron en torno a la tuberculosis en la ciudad de México, a la par de los esfuerzos por edificar instituciones específicamente diseñadas para atender, asistir y "cu-

${ }^{1}$ La historiadora deja muy clara su postura teórica respecto al abordaje que hace de la tuberculosis, basada en los presupuestos presentados por Armus. "alude a diferentes temporalidades, tanto a tiempos largos o, a lo que se podría llamar, "la historia natural de la enfermedad", y a tiempos relativamente más cortos [...] marcados o atados a los avatares generales de una historia necesariamente más específica y acotada a una ciudad o a un país. Esos tiempos más cortos, en ocasiones encuadrados por los quiebres de la historia política, pueden elucidar intervenciones específicas, la "creación y consolidación de agencias estatales", la movilización de nuevas alianzas de sectores sociales y profesionales y la politización de una enfermedad", es decir, su investigación tiende a enunciarse desde un tiempo corto (Agostoni, 2019, p. 521). 
rar" ${ }^{2}$ a los enfermos en el espacio citadino entre el inicio de la década de 1920 y los primeros años de la de 1940. Se centra en mostrarnos los vaivenes por los que pasó la lucha contra la tuberculosis y la materialización de esta en los dispensarios antituberculosos y el Sanatorio para Tuberculosos de Huipulco, de la capital del país, que a decir de la autora "no han ocupado un lugar prominente en la historiografía desde la óptica de la historia social" (Agostoni, 2019, p. 522).

Metodológicamente, nuestra perspectiva para hacer un primer acercamiento a la historia de la tuberculosis está también cercana a la historia de la salud pública (Armus, 2002, 2010). Al respecto, las preguntas que buscamos contestar son: ¿cuándo comenzó a tener relevancia la tuberculosis para las autoridades gubernamentales como problema público?, ¿qué medidas se tomaron?, ¿hasta qué punto han sido efectivas? Pretendemos un conocimiento útil para entender las epidemias y los sistemas sanitarios no como una estampa, sino como una experiencia que pueda brindar destellos de luz sobre la estela de claroscuros que han dejado las enfermedades dentro del territorio bajacaliforniano. ${ }^{3}$ Ahí reside la originalidad de este artículo, en esbozar las características que podría tener un plan para la erradicación de la enfermedad. Por consiguiente, entendemos que los avances de la biomedicina y los análisis químicos contemporáneos nos obligan a generar nuevas interpretaciones sobre el desarrollo de una enfermedad en la sociedad, además de que no todas las acciones que tienen que ver con la vacunación -como la que incluye la vacuna BCG- son acciones de disciplinamiento, "imposiciones saturadas de una suerte de autoritarismo asentado en biopolíticas" (Armus, 2018, p. 840). ${ }^{4}$ Hoy sabemos, por ejemplo, que la tuberculosis es una enfermedad infecciosa

${ }^{2}$ La autora del texto hace énfasis acerca de que "curar" era muy relativo en ese momento y era casi sinónimo de mejora.

${ }^{3}$ Esta investigación no pretende ser una historia sociocultural de la enfermedad, que ha servido como "excusa o recurso para discutir otros tópicos, [porque la historia sociocultural de la enfermedad] apenas dialoga con la historia de las ciencias biomédicas y se concentra en las dimensiones sociodemográficas de una cierta enfermedad" (Armus, 2002, p. 45).

${ }^{4}$ La importancia que la vacuna ha tenido en la medicina es crucial desde que Albert Calmette y Camille Guéran, en 1921, lograron aplicarla con éxito en el hospital la Charité, de París. La vacuna llegó a México en 1925. Fernando Ocaranza, entonces director del Instituto de Higiene, recibió cepas del bacilo. Sin embargo, la vacuna ha sufrido grandes controversias a lo largo de los años. Es bien sabido que en 1929 algunas decenas de niños murieron en una ciudad de Alemania, después de ser vacunados, debido a la "contaminación accidental de la vacuna con una cepa virulenta”, aun así, se comenzó a producir en México desde 1931 hasta 1991 (Carrillo, 2017, pp. 127, 137). 
producida por bacterias del complejo Mycobacterium Tuberculosis, "que puede afectar cualquier órgano del tejido del organismo", se presenta en humanos como en otros mamíferos. Estudios genéticos recientes muestran que existen varias cepas (García, 2013, p. 1).

Aunque nos consideramos asimismo deudores de la llamada Escuela de los Annales, quizá mayormente de sus dos primeras generaciones -representadas por Lucien Febvre, Marc Bloch y Fernand Braudel (Burke, 1999, 2007)-, intentamos pensar la historia de una enfermedad a partir de la dialéctica entre pasado y presente, porque como escribió Marc Bloch (2001): "la incomprensión del presente nace fatalmente de la ignorancia del pasado. Pero quizá es igualmente vano esforzarse por comprender el pasado, si no se sabe nada del presente" (p. 46).

Por lo anterior en este artículo nos proponemos analizar la tuberculosis en Baja California como un proceso aún vigente. A pesar de los avances en la biomedicina y la salud pública, en la actualidad esta entidad ocupa uno de los primeros lugares en incidencia de esta enfermedad, ${ }^{5}$ en lo que respecta sólo a la de tipo pulmonar; que es en la que nos centramos en este texto y la que mayormente ha preocupado a las autoridades gubernamentales (CoPLADE, 2017, p. 30). Aunque se han "identificado" las determinantes sociales ${ }^{6}$ (Coplade, 2017, p. 24) de que existe un tratamiento efectivo y una vacuna inmunizadora, la lucha contra este terrible flagelo ha estado lejos de terminar, como mostramos a continuación.

\section{ANTECEDENTES}

La lucha contra la tuberculosis en Baja California inició durante la segunda década del siglo xx. ${ }^{7}$ Que ello no haya ocurrido antes es una cuestión multi-

${ }^{5}$ La "tasa de morbilidad varía de 40.82 en el año 2000 con 1037 casos a 47.63 con 1684 casos en el año 2016", durante el 2017 el "mayor número de casos se registró en la jurisdicción de Tijuana con 893" (Coplade, 2017, p. 30).

${ }_{6}^{6}$ Las autoridades han señalado en el programa sectorial de salud 2015-2019: "Se trata de un padecimiento asociado a determinantes sociales tales como la marginación, la migración, las comorbilidades y la situación social de los pacientes, lo que dificulta su control e interrupción de la cadena de transmisión" (Coplade, 2017, p. 24).

7 Durante el transcurso de la primera mitad del siglo xx "la тв pulmonar ocupó un lugar prioritario en los programas de salud pública implementados a nivel internacional. En México, de manera similar a cómo aconteció en diferentes países europeos y latinoamericanos, la 
factorial (Fierros, 2014, p. 1): la relativamente reducida población en la entidad; 2) la escasa cantidad de personas aglomeradas en espacios citadinos; 3) el aumento paulatino de la enfermedad en la zona; 4) la falta de sistematización y la estimación de cifras exactas por las autoridades locales, y, como un factor externo, podemos señalar, 5) la falta de herramientas tecnológicas para su detección que sólo pudo concebirse hasta algunos años después de 1882, cuando Robert Koch "identificó al bacilo que lleva su nombre como agente causal de la tuberculosis -y trece años más tarde- Roentgen descubrió los rayos X" (Carrillo, 2012, p. 77), su uso sólo se generalizó tiempo después. Esto ha sido probado en estudios realizados por la historiografía local, que son el antecedente factual más próximo al análisis que realizamos en esta ocasión.

$\mathrm{Al}$ respecto es pertinente mencionar los estudios realizados por el historiador David Piñera $(1991,2006 a)$ que dan cuenta de la situación de las poblaciones bajacalifornianas, donde se observa cómo estas comenzaron a surgir a finales del siglo xIx y con reducidos números de habitantes. Por ejemplo, en 1900 Ensenada -la más importante- tenía sólo 1727 pobladores (Piñera, 2006b, p. 249), el total en Baja California era de 7583 (Piñera, Jaimes y Espinoza, 2012, p. 38).

También están los trabajos realizados por el mismo Piñera y Martínez Zepeda, enfocados a explorar las causas de muerte de las personas de la zona a partir de los registros de defunción, que van de 1901 a 1905. Es importante mencionar que si bien su intención no era analizar la tuberculosis como tal, dan pistas significativas para la comprensión de la enfermedad en los primeros años de Baja California y la tendencia de aumento que tiene hasta nuestros días, pues es de "hacerse notar el elevado número de casos de тв y sus variantes para denominarla". A saber, ni siquiera en los registros oficiales tenían sistematizada la clasificación de la enfermedad misma, como se indica en el texto aludido (Piñera y Martínez, 1994, p. 193). En el periodo mencionado se registraron un total de 53 muertes por tisis pulmonar y 45 por tuberculosis (Piñera y Martínez, 1992, p. 10).

$\mathrm{Al}$ respecto es pertinente precisar que a la tuberculosis se le conoció con distintos nombres: tisis, escrofulosis, la gran plaga blanca, consunción o enfermedad de agotamiento. Hubo varias clasificaciones o formas clínicas, descritas y analizadas a detalle por Manuel Toussaint y Rafael Lavista, mé-

contención de los contagios se sustentó en los dispensarios, los sanatorios y los hospitales" (Agostoni, 2017, p. 200). 
dicos del siglo xIX. Lavista describió las siguientes: la tisis aguda de forma sofocante, la aguda de forma catarral o bronconeumónica y la pleural. Para la clasificación se revisaron las formas anatómicas y clínicas (Guzmán, Salinas y Castañeda, 2013). Lo que puede indicar que ya se tenía una idea de la diferencia de los diversos tipos de la tuberculosis y sus repercusiones, no así la tecnología para estudiarlos como hoy en día.

A partir de los datos y conceptos analizados por las obras referidas, hemos considerado necesario - para efectos de continuidad- revisar el cuatrienio que va de 1906 a 1910 donde encontramos 42 muertes por tisis y 47 por tuberculosis, ${ }^{8} \tan$ sólo nueve muertes menos que en el cuatrienio anterior, explicable en virtud de que durante el año de 1910 hubo un ligero aumento en la tasa de crecimiento de 2.56, es decir, había 9760 personas (Piñera, Jaimes y Espinoza, 2012, p. 41).

La anterior explicación es sustancial para entender por qué la tuberculosis no se convirtió en una problemática para las autoridades gubernamentales de la zona y que no fuera considerada un problema público. Quizá haya habido algunos bandos, reglamentos y tal vez algunas acciones, pero no se organizaron a través de un sistema complejo, como el que se comenzó a formar durante el gobierno de Porfirio Díaz en la capital del país, respecto a diversas cuestiones de salud pública (Agostoni, 2003; Carrillo, 2002). Aunque la introducción de la salud pública en Baja California fue paulatina y se inició durante el porfiriato, no hay pruebas que indiquen que se llevó a cabo una campaña o acciones para el combate de la peste blanca (Fierros, 2014, pp. 41-79), si bien es cierto que en 1907 se lanzó una campaña contra la tuberculosis en la capital del país (Carrillo, 2012), la documentación encontrada hasta ahora nos muestra que no tuvo mucho impacto en Baja California (Fierros, 2014).

El incremento de la importancia de la tuberculosis en la zona también se observa a partir de su morbilidad. En agosto de 1920, Martín Ugalde, enfermero del Departamento de Tuberculosos del Hospital Civil de Mexicali, informó al gobierno que contrajo la enfermedad durante el desempeño de sus funciones. Lo acreditaba mediante un certificado médico expedido por el doctor Manuel Monter. Ugalde solicitaba la cantidad de $\$ 300.00$ oro nacional

${ }^{8}$ Extractos de actas de defunciones del Registro Civil de Ensenada, Tijuana, Mexicali y Tecate de 1906 a 1910. Datos para la historia demográfica de Baja California, exp. 4/8. Copia del Archivo General de la Nación, México, en Acervo Documental del Instituto de Investigaciones Históricas de la Universidad Autónoma de Baja California (en adelante IIH-UABC), México. 
para ir a la ciudad de México a reunirse con sus familiares. La suma se le entregó a manera de "donativo" a través de la tesorería municipal. ${ }^{9}$

Por otro lado, el incremento en la población se hace notar de manera exponencial sólo después de la década de 1920. Había 23537 pobladores con 8.33 de tasa de crecimiento. Hacia 1930, se calcula que había 48 327; en esa década Baja California tuvo una tasa de crecimiento de 8.32 (Piñera, Jaimes y Espinoza, 2012, p. 41).

Sin duda, el aumento de la población y el crecimiento de las ciudades fueron factores que generaron condiciones para que la tuberculosis se desplegara con más facilidad en el territorio, puesto que las cepas del Mycobacterium requieren unas densidades de población elevadas que proporcionen reservas susceptibles para su desarrollo (Porter, 2003, pp. 25-52). A lo que habría que sumarle la dinámica sociocultural de la población derivada de las actividades económicas, por ejemplo, los servicios de corte sexual. Entonces la tuberculosis tomó un papel de mayor importancia para las autoridades locales, que no dimensionaron el problema hasta tiempo después de 1910, cuando comenzaron a aumentar las tasas de mortalidad y morbilidad..$^{10}$ Fue entonces que iniciaron acciones y tomaron medidas en contra de la enfermedad.

\section{LAS PRIMERAS ACCIONES}

La primera y más significativa acción fue la apertura de un lugar para recluir tuberculosos a finales de la década de 1910, en la ciudad de Mexicali, a unas cuadras del Hospital Civil. Contaba con seis camas y una ventanilla por donde se les alimentaba a los ahí recluidos. El lugar era una sección más del Hospital Municipal, ubicado a varios metros, de tal manera que se evitaran las propagaciones de tuberculosis hacia otros enfermos. ${ }^{11}$ Esta acción revela una mayor frecuencia de la enfermedad en la zona.

9 Periódico Oficial del Distrito Norte de la Baja California, Ensenada, Distrito Norte de la Baja California, 5 de agosto de 1920, t. Xxvi, núm. 6, p. 4.

${ }_{10}$ Para dimensionar el asunto, el caso de Tijuana es ilustrativo, lugar en donde tan sólo de 1890 a 1939 murieron 430 personas por тв; de estas sólo 9 fueron registradas antes de 1910 (Mercado, 1986, p. 107).

11 "Los pioneros de la medicina en el Valle de Mexicali", Archivo Histórico del Gobierno del Estado de Baja California (en adelante Aнgeвc), 1910, exp. 23, México. 
Para la década de 1920, la tuberculosis comenzaba a presentar un problema en toda la república mexicana, donde era endémica, "las incompletas cifras recabadas entre 1925 y 1929 [revelan] que por lo menos 1500000 " individuos estaban enfermos (Agostoni, 2019, p. 520). En 1922 "ocupaba el séptimo lugar dentro de las principales causas de mortalidad general" en todo México (Carrillo, 2012, p. 89).

En Baja California hubo grandes preocupaciones. Durante la década de 1920 la tuberculosis había cobrado relevancia en la agenda de los gobiernos locales, tanto que fue motivo para clausurar lugares que permitían la entrada de personas portadoras de la enfermedad. Como ejemplo está el cierre, en 1925, de un templo perteneciente a una iglesia de corte pentecostal en Pueblo Nuevo, Mexicali (Espinoza, 2014, p. 125). Acciones de este tipo las hemos encontrado en Baja California, sin que tengamos referencias de que se dieran también en otras entidades. A inicios de la misma década apareció en el Periódico Oficial un reglamento para la desinfección de casas, que en algún momento fueron ocupadas por personas con tuberculosis.

El reglamento fue revisado por una comisión y aprobado por el Cabildo, entró en vigor el 27 de abril de $1920^{12}$ e incluía once artículos. Llama la atención que en él se contemplaran personas que practicaban la medicina sin título que los avalara como médicos, como se señala en el artículo I. "Toda persona que ejerza la medicina, con autorización o sin ella, está obligado a dar parte por escrito a la Presidencia Municipal, del número de tuberculosos que atienda, el nombre de los mismos, así como el de la calle y el número de la casa que habiten" ${ }^{13}$ La intención era localizar y frenar el avance de la enfermedad de cualquier manera y con ayuda de quien fuera.

Este reglamento era un claro ejemplo de la cooperación en tres sectores distintos de la sociedad: los médicos, el gobierno y los ciudadanos. Los artículos III, VI y VII son muy ilustrativos al respecto:

Art. III. La desinfección deberá hacerse siempre, con conocimiento previo del Médico Municipal, a fin de que se observe en la desinfección los procedimientos prevenidos en este Reglamento. Art. vi. La Autoridad Municipal se reserva

12 Periódico Oficial del Distrito Norte de la Baja California, Ensenada, Distrito Norte de la Baja California, 5 de junio de 1920, t. xxxııI, núm. 31, p. 5.

${ }^{13}$ Periódico Oficial del Distrito Norte de la Baja California, Ensenada, Distrito Norte de la Baja California, 5 de junio de 1920, t. XxxıII, núm. 31, p. 5. 
el derecho de mandar desinfectar las casas que hayan sido ocupadas por pacientes tuberculosos, si después de transcurridos diez días a la desocupación o fallecimiento del paciente o pacientes, no lo verificaren los propietarios o sus representantes, largándoles en el presente caso, el importe de los gastos que la desinfección origine. Art. vir. Para desinfectar una casa se procederá a hacer una fumigación con solución de formaldehida al 10\% p.00; después de esto, las casas empapeladas, serán despojadas de todo su tapiz, cuando esto sea indispensable a juicio del Médico Municipal cuyo tapiz, será desde luego incinerando. A la pared se le dará una encalada, preparando ésta con la misma solución de formaldehida; hecho esto se cerrarán herméticamente todas las rendijas y se sujetará nuevamente a una fumigación de azufre y formaldehida todo esto para cada pieza teniendo cuidado de lo que se acaba de indicar, se practique, no solamente con los muros de la habitación desocupada, sino también con los pisos y techo de las mismas. ${ }^{14}$

El artículo IV hacía referencia a la ubicación de las casas y el registro que debía llevar el Ayuntamiento para poder "notificar oportunamente a los propietarios de las fincas infestadas, que no podrán ser éstas ocupadas nuevamente, mientras no se [ejecutara] la desinfección". ${ }^{15}$

Lo mencionado líneas arriba da cuenta de manera clara del aumento de casos de tuberculosis y la importancia que esta comenzó a tener en Baja California. ${ }^{16}$ Tanto así que se utilizó la prensa como medio de difusión de las noticias relacionadas con la enfermedad. Aunque las acciones preventivas no fueron constantes, debido a que el estado mexicano se encontraba débil e "inmerso en un proceso de reconstrucción y reconfiguración" (Agostoni, 2016, p. 57), no limitó las acciones en contra de la enfermedad, incluso se abrió un lugar a las afueras de las ciudades. No obstante, la apertura de dispensarios antituberculosos en Mexicali y Tijuana se dio hasta finales de la década de los años treinta del siglo $\mathrm{xx}$.

${ }^{14}$ Periódico Oficial del Distrito Norte de la Baja California, Ensenada, Distrito Norte de la Baja California 5 de junio de 1920, t. xxxiII, núm. 31. p. 5.

${ }_{15}$ Periódico Oficial del Distrito Norte de la Baja California, Ensenada, Distrito Norte de la Baja California 5 de junio de 1920, t. xxxiII, núm. 31, p. 5.

${ }^{16}$ Este aumento fue notorio durante la década de 1930, y al parecer, se dio en toda la república mexicana. En 1934 se calcula que la "mortalidad por тв en todo el territorio nacional alcanzó 54.4 defunciones por cien mil habitantes” (Hernández, 1948, p. 706). 


\section{LEJOS DE LA CIUDAD: EL “HOSPITAL" DE LA RUMOROSA}

Durante la década de 1930 las autoridades decidieron enviar los casos más severos de tuberculosis fuera de la ciudad con la intención de evitar la propagación de la misma entre la creciente población de Mexicali, Tijuana, Ensenada, Tecate y otros poblados, de igual forma como aconteció en la ciudad de México (Agostoni, 2019). Para este propósito se eligió un pequeño paraje, situado en la Sierra de Juárez, entre las actuales localidades de Tecate y Mexicali, que años atrás había servido como lugar para centro de gobierno (1924-1929) construido por Abelardo L. Rodríguez, quien denominó a dicho lugar Campo Alaska (Gruel, 2017). Es importante señalar que la elección del sitio obedecía en buena medida a las opciones terapéuticas del momento, que estaban basadas en la benignidad del clima y las condiciones geográficas: "la pureza y sequedad del aire, la temperatura media -nunca extremosa- la protección contra los vientos, la cercanía de bosques y montañas, la luminosidad, la limpieza del cielo y la calma atmosférica". Para favorecer el acceso de los enfermos a aire limpio y los rayos del sol (Agostoni, 2019, p. 527).

En el referido lugar se construyó un hospital ${ }^{17}$ que, durante 27 años, como afirma el sociólogo Manuel Gruel (2017) "no funcionó completamente como psiquiátrico, ni como casa de salud", ya que en distintos momentos, después de su apertura en 1931, se utilizó tanto para albergar personas con diversas enfermedades de tipo psiquiátrico -derivadas de la sífilis en algunos casos-, leprosos y tuberculosos. Posteriormente se remodeló un edificio que albergaba un cuartel militar como un "pabellón para dementes. La casa de gobierno de Abelardo L. Rodríguez era el pabellón antituberculoso”. El número de enfermos de tuberculosis ahí atendidos fue descendiendo, de tal manera de que para 1945 se redujo a 18 (pp. 12, 75, 132, 158).

${ }_{17}$ Antes de 1947, el personal "del hospital se componía de un administrador, dos enfermeras, tres mozos, dos lavanderas e igual número de cocineras". Situación que parece haber cambiado a partir de la fecha aludida pues, "Francisco Díaz Gómez, un estudiante de medicina de la Universidad Autónoma de México; prestador de Servicio Social, mencionó un número diferente: cinco mozos repartidos entre ambos pabellones. Durante las 24 horas del día, cada enfermera, cocinera y lavandera cubría un turno de ocho horas. La misma jornada laboral funcionó para los mozos, quienes se repartieron dos en el pabellón de locos y uno en el antituberculoso" (Gruel, 2017, p. 210). 
En la disminución de la morbilidad probablemente influyó la apertura del dispensario antituberculoso perteneciente al Hospital Civil de Mexicali (нсм) a finales de la década de 1930. En ese entonces, la tuberculosis tenía gran atención por parte de las autoridades federales en todo el país, ya que al exterior de la capital había "18 dispensarios antituberculosos -dependientes ya fuera de los Servicios Sanitarios de los Estados o de los Centros de Higiene Rural-, todos equipados con rayos X y aparatos de neumotórax. Había, asimismo, hospitales especiales para enfermos de tuberculosis, o departamentos específicos para ellos en los hospitales" (Carrillo, 2012, p. 91), lo que refleja el amplio interés mostrado en ese renglón.

En 1942, la Secretaría de Salubridad y Asistencia (ssA) encomendó al médico Gonzalo Espinosa Valle, encargado del dispensario antituberculoso ${ }^{18}$ del Hospital Civil de Mexicali, la tarea de realizar visitas los días domingo al Sanatorio Antituberculoso de La Rumorosa, con el propósito de consultar y vigilar la alimentación de los enfermos. En octubre de 1942 algunos de los que padecían tuberculosis se quejaron del trato que se les daba y, como respuesta a dichas quejas, el gobierno federal envió apoyo; 25 camas y 25 burós sanitarios, además enviaría ropa de cama y otros. Otra de las quejas fue la deficiente alimentación, dicho rubro fue pasado por alto, pues algunos funcionarios consideraban que la cuota que se otorgaba para alimentación era la adecuada, además hubo problemas de corrupción y negligencia (Gruel, 2017, pp. 115, 122, 123). Con falta de fondos e inadecuada atención, la mayor parte del tiempo que estuvo en servicio este lugar funcionó, más como de reclusión que de atención.

En 1943 el gobierno de Mexicali adquirió una camioneta para el traslado de enfermos desde sus hogares hasta el dispensario antituberculoso del HCM en donde se ofrecía consulta externa. Las preocupaciones del gobierno por el crecimiento de la tuberculosis en la zona quedaron en mayor evidencia, cuando en septiembre de 1945 se impartieron pláticas sobre educación higiénica al personal del dispensario y la aplicación de la prueba de Clemens Von Pirquet, con la finalidad de "localizar más enfermos en las colonias de

${ }_{18}$ Aunque en los "dispensarios del Departamento de Salubridad Pública el tratamiento era gratuito, no existía entonces ningún específico efectivo contra la tuberculosis; y se recurría para intentar curarla lo mismo a luz ultravioleta, yodo, oro e inhalaciones de ozono; que a efluvios de alta frecuencia, baños eléctricos y aplicaciones de vacuna de bacilos de tortuga. Como los medicamentos, además de poco efectivos eran muy costosos, se consideraba que lo más práctico era evitar el contagio" (Carrillo, 2012, p. 93). 
Mexicali" (Gruel, 2017, pp. 125, 135). La prueba que consistía en aplicar tuberculina de manera cutánea -extracto proteico obtenido de Mycobacterium- no era eficaz en su totalidad. Si a la persona que se le aplicaba había sufrido infección por sarampión, el virus suprimía la respuesta inmune del huésped (Oldstone, 2002, p. 106).

Las acciones realizadas hasta principios de la década de 1940 por las autoridades locales, que en ocasiones se ayudaron de fondos federales, parecieron ser insuficientes para detener la propagación y la consecuente endemicidad de la tuberculosis en Baja California. En ese año, Lázaro Cárdenas "le solicito al Departamento de Salubridad Pública un programa de higienización de los territorios, para controlar en especial las enfermedades endémicas, sobre todo el paludismo" y la misma tuberculosis (Cruz, 2004, p. 45). El control de las mencionadas enfermedades se incluyó en el Programa de Cooperación Interamericana de Salubridad (PCIS) en la Frontera Norte de la República Mexicana, que tenía como finalidad "el incremento de algunas de las actividades de carácter preventivo a cargo de las Oficinas Sanitarias en las poblaciones situadas a lo largo de la línea divisoria con los Estados Unidos” (Hernández, 1948, p. 706).

\section{EL PROGRAMA DE COOPERACIÓN INTERAMERICANA DE SALUBRIDAD}

Para 1941 Baja California ya formaba parte de este programa, aunque su situación era bastante precaria debido a que no había los fondos suficientes. El programa contaba con "unidad fotofluorografía, transportable, de tipo pesado, para la toma de películas de 35 milímetros" (Hernández, 1948, p. 710) que recorría algunos poblados pertenecientes a estados que se encontraban en la franja fronteriza. A pesar de poseer tecnología de punta -en número insuficiente para abarcar los poblados fronterizos- en la zona no se tenía ninguna institución especializada en tuberculosis, tampoco un "hospitalario o sanatorial para internar enfermos" (Hernández, 1948, p. 711). La preocupación por el combate de esta enfermedad en el territorio bajacaliforniano parece tener su fundamento en las cifras sobre mortalidad arrojadas por epidemiólogos adscritos al programa, que señalaban y comparaban el coeficiente que se presentaba en Baja California (182.3) con el de otros estados fronterizos como "Sonora 132.8, Chihuahua 60.9, Coahuila 118.4, Nuevo León 132.2 y Tamau- 
lipas 134.1" por cada 100000 habitantes, donde Baja California ocupaba el primer lugar, lo mismo sucedía con los municipios; Mexicali fue el más alto con 214.7 por cada 100000 habitantes (Hernández, 1948, p. 709).

No cabe duda de que la erradicación de la tuberculosis, no sólo de Baja California sino de toda la región fronteriza, se veía como una misión imposible (Hernández, 1948). Por eso el PCIs intentaba cumplir con tres puntos básicos a través de los subcomités que se formaron a lo largo de la frontera:

En primer lugar, la parte que corresponde a Educación Higiénica en relación con $\mathrm{TB}$, procurando hacer amplia difusión popular de conocimiento sobre la manera como se contagia la тв, cómo se previene, las consecuencias inmediatas de la enfermedad también factores económicos y sociales [...] En segundo lugar, la promoción e impulso de la cooperación privada para la resolución de los diversos problemas que afloran como consecuencia de la influencia nociva que el enfermo tuberculoso ejerce sobre el medio social [...] En tercer lugar, la promoción e impulso de todas las actividades dentro del medio social que tiendan a la mejor colaboración mutua entre, las instituciones oficiales y privadas que en alguna forma participan en la lucha contra la тв (Hernández, 1948, pp. 712-713).

Las acciones de salud pública que se generaron a partir del Pcis en contra de la tuberculosis estuvieron limitadas por la falta de recursos económicos, materiales y humanos. Aunque hubo algunas acciones donde se involucraron los tres órdenes de gobierno e incluso la sociedad civil. La problemática para la correcta coordinación de servicios de salud pública se debía en parte a la falta de vías de comunicación entre Baja California y el "interior del país” (Cruz, 2004, p. 44), como había señalado el presidente Lázaro Cárdenas durante su mandato.

A propósito de la ubicación geográfica de Baja California, cabe resaltar su condición fronteriza, ya que desde finales del siglo xix el gobierno estadunidense comenzó a realizar acciones de salud pública en la frontera entre México y Estados Unidos (Fierros, 2016). Esta condición fue un elemento relevante en la lucha contra la tuberculosis pues, en 1946, autoridades de la American Public Health Association presentaron un informe de salud pública sobre la ciudad y el condado de San Diego, California. En él señalaban que hasta ese momento la mayoría de las acciones para el control de la tuberculosis se habían llevado a cabo por la Tuberculosis Association que era una 
organización altruista y se movía por el apoyo del voluntariado y, en menor medida, por el Public Health Nursing Service (Buck y Kandle, 1946, p. 4). En el mencionado informe entendían que la tuberculosis y algunas enfermedades venéreas deberían ser tratadas de manera inmediata por el Departamento de Salud, así como la vigilancia de las zonas aledañas al condado, que pudieran ser focos de infección, a decir las poblaciones de Baja California (p. 44).

Del lado mexicano la falta de recursos dificultaba la lucha contra la tuberculosis, el médico Ricardo Hernández Ballados (1948), participante en el programa, admitió: "No pretendemos erradicar la tuberculosis entre nosotros a plazo breve porque sería una tarea para la cual, aun cuando nos sobra voluntad y ponemos en ella todos nuestros esfuerzos, nos faltan elementos materiales con los cuales poder aspirar a ese objetivo" (p. 713). Sus expectativas eran realistas, pues fue hasta 1950 que la lucha contra la tuberculosis "se fortaleció con la llegada al país [...] de tratamientos efectivos: antibióticos y antifímicos específicos, que dieron confianza a los tisiólogos en la posibilidad de erradicar la enfermedad. A partir de ese momento cambió la política de control de la enfermedad, y el tratamiento de la тв pasó a ser básicamente ambulatorio" (Carrillo, 2012, p. 94). Hernández Ballados (1948) era consciente de las condicionantes económicas al expresar: "nos conformamos por ahora con mejorar nuestras escasas instituciones, crear aquellas que nuestra posibilidad económica nos permita y nos conformaremos con disminuir nuestros coeficientes de mortalidad hasta lograr que, por lo que corresponde a la TB, ésta deje de ser el factor tan importante que es en nuestra epidemiología” (p. 713), por lo que es necesario hacer mención que entre 1939 y 1950 las ofertas de la biomedicina para curar la tuberculosis eran "inciertas” (Agostoni, 2017, p. 201).

\section{DESPUÉS DE LOS TRATAMIENTOS}

Historiadores, epidemiólogos y expertos en salud pública coinciden en que después de la década de 1950 el combate contra la tuberculosis en México fue favorecido cuando la quimioterapia, la vacuna BCG y los antibióticos "modificaron las estrategias" para prevenirla y curarla (Agostoni, 2017, p. 200). Aunque entre finales de la década de 1940 y principios de la 1950 se había descubierto la estreptomicina -por Waskman en 1945-, el ácido paraaminosalicítico -por Lehman en 1946- y la hidracida del ácido isonicotínico -por Robitzek y Selikof en 1952-, es casi seguro que su uso se generalizaría entre la sociedad 
civil tiempo después de su descubrimiento. El uso de estos antibióticos fue paradójico, pues aunque ayudaron en atenuar el problema, enseguida aparecieron cepas resistentes (Ferreira y Velázquez, 1993, p. 98; Porter, 2003, p. 171).

La vacuna dio esperanzas de anulación, pero aun así, en Baja California la enfermedad seguía presentado cifras ascendentes en su morbilidad, tan sólo de 1946 a 1949 se infectaron 1769 y de 1949 a 1951 lo hicieron 3940 (Gruel, 2017, p. 145). No es de sorprendernos que las cifras hayan ido en aumento para el trienio que va de 1951 a 1953 con 4092 infectados (Gruel, 2017, p. 145), ya que durante esos años hubo un aumento de población. En 1940 había 78907 habitantes y para 1950 aumentó a 226 956, pues de hecho en este año se dio la tasa de crecimiento más alta del siglo $\mathrm{xx}$, con $11.14 \%$ (Piñera, Jaimes y Espinoza, 2012, p. 41). Si se consideran las cifras porcentualmente, vemos que en esos años hubo una reducción en la morbilidad. Los programas preventivos habían tenido cierto margen de éxito, porque este no es un indicador de personas recuperadas por la aplicación de antibióticos.

Pero la vacuna contra la tuberculosis no llegó a Baja California -al menos de manera oficial- sino hasta los primeros años de la década de los cincuenta mediante campañas de vacunación. Esto fue así pues aunque la campaña contra la tuberculosis que había incluido la vacunación con BCG se inició en una parte de la república mexicana "desde la década de 1930" (Carrillo, 2017, p. 130), en la zona no había suficientes recursos humanos y materiales para brindar la atención médica que demandaba la creciente población, ni hablar de organizar una campaña de vacunación. Es verdad que en 1941 se creó el Hospital General de Tijuana ${ }^{19}$ pero las polémicas entorpecieron la difusión de la vacuna porque

en 1950 varios bebés recién nacidos, vacunados con BCG oral durante los primeros diez días de vida, murieron de tuberculosis generalizada [...] La vacunación oral se suspendió, como en la mayoría de los países en ese momento, pero la controversia también afectó la vacunación intravenosa. Los que se oponían a la BCG oral sugirieron métodos de control aprobados por la experiencia, y los defensores de la vacunación contra la tuberculosis tardaron diez años en poder regresar la vacuna a México (Carrillo, 2017, p. 130).

${ }^{19}$ Manuel Ávila Camacho, exp. 562.11/42 ADD. IIH-UABC. 
La realidad es que había pocos recursos médicos y paramédicos hasta para la instalación de hospitales pertenecientes al Instituto Mexicano del Seguro Social (Olvera, 2014, p. 16) que inclusive hace algunos años tuvieron un papel preponderante en las campañas de vacunación. El retraso de la llegada de la vacuna a la zona se vio obstaculizado por falta de organización. En Baja California este proceso se intensificó a finales de la década de los setenta y principios de la de los ochenta, como consecuencia del Programa Nacional de Inmunizaciones de 1973. Otra de las consecuencias fue la aparición de la cartilla de vacunación en 1979 (LatinComm, 2015) que es hasta nuestros días un requisito para el ingreso de los niños a los niveles preescolar y primaria (ya sea pública o privada).

Posteriormente se organizó una campaña de vacunación masiva mediante el Programa Nacional de Inmunizaciones, en el que se establecía la aplicación de cuatro vacunas obligatorias, entre las que estaba la BCG (las otras eran antipoliomielítica, DPT, antisarampión y el toxoide tetánico). "En apoyo a este programa, desde 1980 se organizaron jornadas intensivas de vacunación con características y denominación diversas pero con objetivos similares": primero fueron las Fases Intensivas de Vacunación, después los Días Nacionales de Vacunación, que posteriormente se nombraron Semanas Nacionales de Vacunación y, finalmente, Semanas Nacionales de Salud. En 1985 se publicó un decreto que establece como obligatoria la Cartilla Nacional de Vacunación y, en un esfuerzo por la unificación de los programas, en 1991 se creó el Programa de Vacunación Universal (Hurtado y Matías, 2005, p. 50).

El aumento de las cifras comenzó a representar un problema binacional entre México y Estados Unidos, desde finales de la década de 1930, pero el énfasis en la tuberculosis se incrementó a partir de la década de 1950, cuando en ambos lados de la frontera se crearon programas de control que tenían un enfoque transfronterizo. Uno de ellos fue el CURE TB, operado por el Programa de Control de Tuberculosis del condado de San Diego, California, iniciado en la década aludida (Organización Panamericana de la Salud, 2011, p. 63).

Además, se realizaron estudios en la zona por expertos de ambos lados, muchos de ellos auspiciados con fondos federales. Un ejemplo de esto es el que se llevó a cabo en Mexicali y el área rural, efectuado mediante un convenio entre la ssA, la Organización Mundial de la Salud (OMS) y el Fondo de las Naciones Unidas para la Infancia (UNICEF). En este programa, tan sólo en el municipio de Mexicali, de 1962 a 1963 se examinó con fotofluorográfía a 7304 personas, de las cuales 132 dieron positivo (Izaguirre, Blancarte y Santos, 1964, p. 798). 
La aplicación de antibióticos generó un descenso de $50 \%$ en la mortalidad, pues entre 1963 y 1974 hubo 2927 muertes ocasionadas por la tuberculosis, según informó Enrique Verduzco (1980), asesor del Departamento de Enfermedades Crónicas y Degenerativas de la Jefatura de Servicios de Medicina Preventiva, del Instituto Mexicano del Seguro Social (IMss). Aun así, los tratamientos eran poco efectivos, aunque se habían descubierto otros antibióticos como el etambutol en 1969, ya que había escaso seguimiento por parte de los pacientes. Durante mayo de 1974, de las 314 personas detectadas con tuberculosis, sólo 73 dieron seguimiento de manera correcta (Sentíes, Ortiz, Bonilla, Rodarte y Escobedo, 1974).

Fue precisamente en el año mencionado que padecer tuberculosis apareció en el Código Civil para el estado de Baja California (artículo 264) como causa de divorcio. Años más tarde se realizó una reforma al artículo 94, en donde se indicaba que para contraer matrimonio era necesario presentar un certificado suscrito por un médico titulado, en donde se asegurara la ausencia de tuberculosis. Es importante enfatizar esta cuestión, pues hoy en día ya no se exige este certificado, sólo una prueba que descarte enfermedades de transmisión sexual..$^{20}$

A pesar de lo anterior, a finales de la década de los setenta y principios de la de los ochenta, se llegó a creer que la tuberculosis podía controlarse y erradicarse debido a los métodos de detección, los tratamientos y las campañas de vacunación masiva. Estas últimas siguieron durante las décadas subsecuentes, "a partir de 1971, comenzó la producción de la forma liofilizada de esta vacuna en el Instituto Nacional de Higiene, usando la cepa Danesa 1331" (LatinComm, 2015).

En este contexto y debido a un programa de control inadecuado, la resistencia a los fármacos antituberculosos ha aumentado. [...] en 1976 reportaron una resistencia inicial a la isoniacida y estreptomicina del 3.9 y $14.3 \%$, respectivamente. Ya, en 1982 [se] reportó 6.6\% de resistencia inicial a la isoniacida, 0.8\% a la rifampicina y $15.4 \%$ a la estreptomicina. En 1995, [se] informó $9 \%$ a isoniacida, $2 \%$ a rifampicina y $12 \%$ a estreptomicina. Por último en el Proyecto de Vigilancia de Resistencia a Fármacos Antituberculosos, [ha encontrado] resistencia en tres estados mexicanos (Baja California, Sinaloa y Oaxaca), reportaron que

${ }^{20}$ Periódico Oficial del Estado de Baja California, Mexicali, Baja California, 31 de enero de 1974, t. LXXXI, núm. 3. 
la resistencia en casos nuevos y retratados a la Isoniacida fue del 12.9 y $50.5 \%$ respectivamente, para tasas de multifármacorresistencia de 2.4 y del $22.4 \%$, lo que habla de que en nuestro país también existen altos niveles de resistencia (Villalba, 2003, p. 487).

Contrariamente de los esfuerzos, aún hacia 1991 Baja California figuraba entre los estados con mayor índice de mortalidad, acompañado de Chiapas, Veracruz y Oaxaca. Para esa década las preocupaciones habían aumentado de manera exponencial, durante 1999 se llevaron a cabo varias reuniones para tomar acciones conjuntas entre autoridades de México y Estados Unidos en contra de la tuberculosis (Organización Panamericana de la Salud, 2011, p. 11). Por su parte la Comisión de Salud Fronteriza México-Estados Unidos resaltó en 2003, "en México la tasa de incidencia nacional de la tuberculosis disminuyó en un 15 por ciento entre 1995 y 2000, mientras que en los estados fronterizos sólo disminuyó un cinco por ciento, su tasa de incidencia es significativamente más alta que la nacional pues la tasa nacional fue de 15.7 mientras que en los municipios fronterizos la tasa fue de 33.4 casos por cada 100,000 habitantes en el año 2000" (López, 2014, p. 33).

Para contrarrestar los embates de nuevos ataques de cepas de tuberculosis resistentes a los antibióticos, en 2010 se creó la Iniciativa diez contra la TB, planteada desde 1995. La enfermedad parece causar más que preocupaciones y mover la burocracia médica de ambos lados de la frontera. A través de esta se propuso "identificar y responder a las oportunidades y retos que no pueden ser solucionados de manera unilateral por ninguno de los países actuando separadamente". Fueron necesarios "esfuerzos binacionales de cooperación a lo largo de la frontera, con el propósito de reducir la morbilidad y mortalidad a causa de esta enfermedad y reducir su transmisión" (Organización Panamericana de la Salud, 2011, p. 62). Las cosas parecen estár lejos de terminar así, pues día con día hay un tráfico exagerado de personas entre los dos países.

A inicios del siglo xxi Baja California "ocupa el primer lugar a nivel nacional en mortalidad por тв; en el año 2000 se registraron 157 defunciones con tasa de mortalidad de 6.57 y en 2016 se presentaron 241 defunciones y tasa de 6.82 por cada 100 mil habitantes". Esta situación ha sido señalada como un asunto prioritario por las autoridades estatales, han enfatizado que se requiere "la implementación de políticas públicas que garanticen su atención integral por sus connotaciones sociales, culturales y económicas" (CoPLADE, 2017, pp. 30-31). Aun así, no se ha visto la atención en esta área de la salud. 


\section{UNA CUESTIÓN POR DEFINIRSE}

La lucha contra la tuberculosis en Baja California está lejos de acabar. Las dinámicas sociales, demográficas, económicas e incluso culturales de la población parecen ser las causas en las que se encuentra la respuesta para dar fin a la enfermedad. No es casual que un médico mexicano a inicios del siglo xx escribiera -en tono profético-: "a pesar de todos los esfuerzos de los terapeutistas y de los higienistas, el problema de la tuberculosis sigue siendo tan pavoroso como antes" (Carrillo, 2012, p. 89). Porque, en efecto, la tuberculosis no es una enfermedad "infecto-contagiosa reemergente", como indican las autoridades gubernamentales (COPLADE, 2017, p. 7), sino una enfermedad que ha habitado en la zona por más de 100 años y se ha presentado en estado latente y constante. Nunca desapareció y como se observa ha incrementado su prevalencia con el paso de los años. Sobre todo cuando, en 2015, se encontraba en el número catorce de las principales causas de mortalidad en Baja California, con 211 decesos y una prevalencia de 6.06 (Coplade, 2017, p. 27).

Proyecciones alentadoras de las autoridades gubernamentales indican que para 2030 esperan poner fin a la "epidemia" de tuberculosis, además de la de SIDA (COPLADE, 2017, p. 12) en donde sólo parecen estar involucradas dos instituciones de salud, IsESALUd e IssSTECALI (p. 51), cuando hoy en día Baja California cuenta con más de 3000000 de habitantes y la realidad es que hay pocos espacios para brindar dicho servicio al total de la población. Estamos en espera de una bala mágica que sea rápida o un programa de salud pública realmente efectivo. A diferencia de enfermedades como el sIDA, la tuberculosis tiene cura, si se detecta en sus etapas tempranas.

La vacunación ha sido un buen recurso para evitar muertes prematuras por la enfermedad. La aplicación de la vacuna durante los últimos quince años ha tenido gran impacto, "el mayor número de casos de тв se presentó entre los años 2004 y 2006, con 6, 9 y 5 respectivamente”. En 2016 se registró sólo un caso (Coplade, 2017, p. 12), es decir, la vacuna funciona solamente con ciertos grupos, cuando los organismos son receptores; no obstante, para que funcione como método profiláctico en el total de la población y tenga un impacto a gran escala, se requiere crear un programa que sea integral, que considere las dinámicas sociales, demográficas, económicas y culturales propias de las ciudades de Baja California.

Consideramos que el programa integral deberá tener en cuenta como asuntos prioritarios en las políticas públicas los siguientes aspectos: 1) el cre- 
cimiento acelerado y en buena medida carente de organización que registran, a partir de los años ochenta del siglo xx, las ciudades bajacalifornianas, especialmente Tijuana y Mexicali. En la primera de ellas la situación es crítica, dada la proliferación de conjuntos habitacionales causantes del hacinamiento de personas. En Mexicali, la mayoría de la población se concentra en un espacio reducido del área urbana (Flores, 2015). 2) La situación fronteriza de la entidad, que atrae del interior del país flujos migratorios incidentes en problemas demográficos antes aludidos y que son proclives a la propagación de la tuberculosis. Por último, 3) los factores ambientales a los que están expuestos los enfermos de VIH, especialmente susceptibles a la enfermedad (França y Ferreira, 2013).

En el plan integral que sugerimos han de contemplarse acciones que consideren los tres aspectos señalados, interrelacionándolos debidamente, a fin de que las medidas sanitarias de higiene pública que se tomen alcancen un sentido de integralidad.

\section{LISTA DE REFERENCIAS}

Agostoni, C. (2003). Monuments of progress modernization and public health in Mexico City 1876-1910. Calgary: University of Calgary Press.

Agostoni, C. (2016). Médicos, campañas y vacunas. La viruela y la cultura de su prevención en México, 1870-1952. México: Instituto de Investigaciones Históricas-unAM.

Agostoni, C. (2017). 'Timbres rojos' y el Comité Nacional de Lucha contra la Tuberculosis, Ciudad de México, 1939-1950, Revista CONAMED, 22(4), 199-201. Recuperado de https://www.medigraphic.com/cgi-bin/new/resumen.cgi?IDARTICU$\mathrm{LO}=79270$

Agostoni, C. (2019). Del "armamento antituberculoso" al Sanatorio para Tuberculosos de Huipulco en la Ciudad de México, 1920-1940. História, Ciências, Saúde Manguinhos, 26(2), 519-536. DoI: https://doi.org/10.1590/s0104-59702019000200009

Armus, D. (2002). La enfermedad en la historiografía de América Latina moderna. Asclepio, 54(2), 41-60. DoI: https://doi.org/10.3989/asclepio.2002.v54.i2.140

Armus, D. (2010). ¿Qué historia de la salud y la enfermedad? Salud Colectiva, 6(1), 5-10. Recuperado de https://www.scielosp.org/j/scol/i/2010.v6n1/

Armus, D. (2018). Sobre Claudia Agostoni, Médicos, campañas y vacunas. La viruela y la cultura de su prevención en México 1870-1952, Historia Mexicana, 68(2), 838841. DoI: http://dx.doi.org/10.24201/hm.v68i2.3596 
Bloch, M. (2001). Apología para la historia o el oficio de historiador. México: Fondo de Cultura Económica.

Buck, C. y Kandle, R. (1946). Public health in San Diego County and City. Nueva York: American Public Health Association.

Burke, P. (1999) La revolución historiográfica francesa. La escuela de los Annales: 19291989. Barcelona: Gedisa.

Burke, P. (2007) Historia y teoría social. Argentina: Amorrortu.

Carrillo, A. M. (2002). Economía, política y salud pública en el México porfiriano (1876-1910). História, Ciências, Saúde Manguinhos, 9(suplemento), 67-87. DoI: http://dx.doi.org/10.1590/S0104-59702002000400004

Carrillo, A. M. (2012). Los modernos minotauro y Teseo: la lucha contra la tuberculosis en México. Estudios, número especial, 85-101. DoI: https://doi.org/10.31050/ re.v0i0.2556

Carrillo, A. M. (2017). Vaccine production, national security anxieties and the unstable State in nineteenth and twentieth century Mexico. En: C. Holmberg, S. Blume y P. Greenough, The politics of vaccination: a global history (pp. 121-147). Manchester: Manchester University Press.

Coplade (Comité de Planeación del Desarrollo) (2017). Programa sectorial de salud 2015/2019. Recuperado de http://www.copladebc.gob.mx/programas/sectoriales/ Programa\%20Sectorial\%20de\%20Salud.pdf

Cruz, N. (2004). Baja California en el contexto de la política de población durante el periodo cardenista, 1930-1940. (Tesis de maestría). El Colegio de la Frontera Norte, Tijuana, Baja California.

Espinosa E., Paredes F. y Málaga A. (1959). Tuberculosis bovina en México. Boletín de la Oficina Sanitaria Panamericana, 46, 136-139.

Espinoza, P. (2014). La Iglesia católica ante el Estado posrevolucionario en Tijuana, 19261935. (Tesis de maestría). Universidad Autónoma de Baja California, Tijuana, Baja California.

Ferreira, E. y Velázquez, O. (1993). Tuberculosis en México: otro emisario del pasado. Boletín Mensual de Epidemiologia, 7, 97-111.

Fierros, A. (2014), Historia de la salud pública en el Distrito Norte de la Baja California 1888-1923. México: ConAculta.

Fierros, A. (2016). Los inicios de la salud pública en Baja California, México, y California, Estados Unidos, siglos xIx y xx. Región y Sociedad, 28(67), 315-346. DOI: https://doi.org/10.22198/rys.2016.67.a805

Flores, G. (2015). Proyecciones de población urbana y rural de las localidades de Baja California 2015-2030. Recuperado de http://www.copladebc.gob.mx/publicacio- 
nes/2015/Proyecciones\%20de\%20poblacion\%20urbana\%20y\%20rural\%20de\%20 las\%20localidades\%20de\%20Baja\%20California\%202015-2030.pdf

França, N. y Ferreira, M. (2013). Los factores sociales y ambientales asociados a la hospitalización de los pacientes con tuberculosis. Revista Latino-Americana de Enfermagem, 21(2), 1-8. DoI: https://doi.org/10.1590/S0104-11692013000200006

Gruel, M. (2017) Rumor de locos El Hospital de La Rumorosa, 1931-1958. México: Instituto Sudcaliforniano de Cultura.

García, A. (2013). Epidemiologia molecular de la tuberculosis en Baja California. (Tesis de maestría). Universidad Autónoma de Baja California, Ensenada, Baja California.

Guzmán, S., Salinas, C. y Castañeda, G. (2013). La tuberculosis en México: aportaciones del Museo Anatomopatológico, 1895-1899. Revista de Investigación Clínica, 65(1), 94-101. Recuperado de https://www.medigraphic.com/cgi-bin/new/resumen.cgi?IDARTICULO $=42463$

Hernández, R. (1948). Programa de lucha antituberculosa en la frontera norte de México. Boletín de la Oficina Sanitaria Panamericana, 19, 706-713.

Hurtado, C. y Matías, N. (2005). Historia de la vacunación en México. Revista Mexicana de Puericultura y Pediatría, 13(74), 47-52.

Izaguirre, A., Blancarte, R. y Santos, C. (1964). Panorama epidemiológico de la tuberculosis pulmonar en México. Salud Pública de México, 5, 787-801.

LatinComm (2015). Historia y avances de la vacunación en México. Recuperado de http://www.latincomm.com/

López, A. (2014). Los pacientes con VIH/sIDA en Tijuana y la accesibilidad a los servicios de salud como factor determinante de la adherencia terapéutica. (Tesis de doctorado). El Colegio de la Frontera Norte, Tijuana Baja California.

Mercado, R. (1986). Los pioneros de la medicina en Tijuana. México: Litografía Rivera. Oldstone, M. (2002). Virus, pestes e historia. México: Fondo de Cultura Económica. Olvera, A. (2014). El seguro grande. El Río, 24, 15- 17.

Organización Panamericana de la Salud (2011). Situación de la tuberculosis en la frontera México-Estados Unidos. El Paso Texas: Organización Panamericana de la Salud/ Organización Mundial de la Salud.

Piñera, D. (1991). Los orígenes de Ensenada y la política nacional de colonización. México: Universidad Autónoma de Baja California.

Piñera, D. y Martínez, J. (1992). Datos para la historia demográfica de Baja California. Causas de defunción: 1901-1905. Tijuana: Instituto de Investigaciones Históricas-UABC.

Piñera, D. y Martínez, J. (1994). Baja California 1901-1905. Consideraciones y datos para su historia demográfica. Tijuana: UABC /UnAM/sDsu. 
Piñera, D. (2006a). Los orígenes de las poblaciones de Baja California. Factores externos, nacionales y locales. México: UABC.

Piñera, D. (2006b). Las compañías colonizadoras y los orígenes de las poblaciones. En M. Samaniego, Breve historia de Baja California (pp. 99-124). Mexicali: Universidad Autónoma de Baja California.

Piñera, D., Jaimes, R. y Espinoza, P. (2012). Trayectorias demográficas de Baja California y California, 1900-2000. Contrastes y paralelismos. Estudios Fronterizos, 13(26), 33-61. DoI: https://doi.org/10.21670/ref.2012.26.a02

Porter, R. (2003). Breve historia de la medicina desde la antigüedad hasta nuestros días. México: Taurus.

Sentíes, R., Ortiz, A., Bonilla, J., Rodarte, H. y Escobedo, A. (1974). Bases del programa de control de la tuberculosis en México. Boletín de la Oficina Sanitaria Panamericana, 4, 479-493.

Verduzco, E. (1980). La mortalidad por tuberculosis en la República Mexicana durante el periodo 1963-1974. Salud Pública de México, 2, 191-228.

Villalba, J. (2003). Tuberculosis: un problema de actualidad. Gaceta Médica de México, 139(5), 471- 492.

\section{OTRAS FUENTES}

Archivos

IIH-UABC Instituto de Investigaciones Históricas de la Universidad Autónoma de Baja California. Copias de acervo documental del Archivo General de la Nación, México.

Ahgebc Archivo Histórico del Gobierno del Estado Baja California. 